\title{
Large, Spontaneous Macular Hole with Posterior Pole Detachment in a Patient with Best Vitelliform Macular Dystrophy
}

\author{
Shamfa Peart ${ }^{\mathrm{a}} \quad$ Amoy Ramsay $^{\mathrm{b}} \quad$ Qazi Assad Khan $^{\mathrm{c}}$ Tony Leong ${ }^{\mathrm{c}}$ \\ Patel Gordon-Bennett ${ }^{\mathrm{c}}$ \\ aDepartment of Ophthalmology, University Hospitals of Leicester NHS Trust, Leicester, UK; \\ bDepartment of Ophthalmology, Cornwall Regional Hospital, Montego Bay, Jamaica; \\ 'Department of Ophthalmology, Western Sussex Hospitals NHS Trust, Worthing, UK
}

\section{Keywords}

Macular hole $\cdot$ Best macular dystrophy $\cdot$ Posterior pole detachment $\cdot$ Silicone oil

\begin{abstract}
Purpose: To describe the visual and anatomical outcomes in a patient with a full-thickness macular hole and Best vitelliform macular dystrophy. Methods: The authors present a case of a large spontaneous macular hole with associated posterior pole detachment in a patient with a history of Best vitelliform macular dystrophy including clinical course and surgical outcome. Patient: The patient presented with a history of blurred central vision. He was known to have Best vitelliform macular dystrophy. Examination revealed BCVA $6 / 36$ (0.78 logMAR) and a fullthickness macular hole $(1,102 \mu \mathrm{m})$ with a shallow posterior pole detachment extending to the vascular arcades. He underwent phacovitrectomy with silicone oil tamponade. Internal limiting membrane (ILM) peel was prohibited due to a very adherent posterior hyaloid membrane (PHM). Results: The patient developed type 2 closure. He had oil removal in 14 months combined with PHM and ILM peel. Two months postoperatively, he had further reduction of the foveal defect and the retina remained flat. Final BCVA was $6 / 24$ (0.60 logMAR). Conclusion: Macular holes with Best disease are rare and are thought to be due to rupture of a cyst in the vitelliform stage or atrophy in later stages. This case outlines that closure of the macular hole, flattening of the detachment, and improvement in visual acuity is possible with vitrectomy and ILM peeling.




\section{Introduction}

We report a case of a large, spontaneous macular hole associated with a posterior pole retinal detachment in a middle-aged male with a history of Best vitelliform macular dystrophy. The patient provided written informed consent for the publication of this material.

\section{Case}

A 51-year-old male presented with a history of blurred central vision to the left eye for 3 weeks. He described it as an oil droplet appearing in his central vision. He reported difficulty in performing close tasks which he was able to do premorbidly. His left eye was his better eye.

He had a history of Best vitelliform macular dystrophy, which had been diagnosed with electrodiagnostic tests in childhood at a tertiary eye centre. Genetic analysis was not done at the time. His mother was also affected but his sister was unaffected. He denied previous trauma or surgery and was bilaterally emmetropic. He was otherwise fit and well.

On examination, his best-corrected visual acuity was ${ }^{6} / 60(1.00 \log \mathrm{MAR})$ on the right and $6 / 36$ (0.78 logMAR) on the left. His intraocular pressures were 28 and $33 \mathrm{~mm} \mathrm{Hg}$ on the right and left, respectively. His anterior segment was normal bilaterally with mild nuclear sclerosis. There was no posterior vitreous detachment and no associated tobacco dust. His optic discs were healthy with cup:disc ratios of 0.4 on the right and 0.45 on the left. He had pigmentary foveal changes on the right side with macular scarring (Fig. 1a). On the left, he had a large macular hole measuring $1,102 \mu \mathrm{m}$ with a shallow posterior pole retinal detachment extending to the vascular arcades (Fig. 1b, Fig. 2a). No peripheral retinal breaks were detected. He had left macular pigmentary changes as well. The vitelliform lesions were stage 5 (atrophic) bilaterally.

He was commenced on latanoprost drops to both eyes at night to control the elevated intraocular pressures. The patient agreed to having combined phacoemulsification and intraocular lens implant with pars plana vitrectomy, internal limiting membrane (ILM) peel and silicone oil, followed by prone positioning for 7 days.

During surgery, a gelatinous and very adherent posterior hyaloid membrane (PHM) was found, which was difficult to remove and prohibited ILM peel. On the first postoperative day, the patient noticed a subjective improvement in his vision. His uncorrected left visual acuity was $6 / 60(1.00 \operatorname{logMAR})$, with flat retina and good oil fill.

Two weeks postoperatively, the visual acuity was $6 / 60$ (1.00 logMAR) improving to $6 / 36$ ( $0.78 \log$ MAR $)$ with pinhole. At 3 months, his pinhole acuity improved to $6 / 24$ ( 0.60 logMAR) and the retina remained flat. OCT of the macula showed that the hole had closed (type 2 closure) but there were central areas of photoreceptor layer atrophy (Fig. 2b).

Eleven months later, his retina remained flat and the macular hole closed (Fig. 2c). At 14 months, removal of silicone oil was performed along with PHM peel and ILM peel as a single procedure. Two weeks post-silicone oil removal and ILM peel, the retina remained flat and the patient's unaided left vision was $6 / 24(0.60 \operatorname{logMAR})$. The type 2 closure of the macular hole also improved with reduction in the foveal defect from $637 \mu \mathrm{m}$ to $254 \mu \mathrm{m}$ after oil removal and ILM peel (Fig. 3). 


\section{Case Reports in Ophthalmology}

\section{Discussion}

The pathogenesis of macular hole and retinal detachment formation in Best dystrophy remains unclear [1]. Several factors have been postulated including rupture of a cyst in the vitelliform stage and atrophic changes in the neurosensory retina and retinal pigment epithelium in the late stages [2-4]. Histopathological analysis suggests that Best dystrophy is due to a generalised abnormality of the retinal pigment epithelium, which leads to the accumulation of abnormal fibrillar material and lipofuscin granules [3,5]. Tangential vitreous traction has also been hypothesized as a pathogenetic mechanism as relieving this traction with vitrectomy has led to the closure of the macular holes in some cases [6]. It is noteworthy that these patients tend to be emmetropic [3].

The development of retinal detachment in Best macular hole has also been explained. Reduction in the pumping effect of the dysfunctional retinal pigment epithelium impairs its dehydrating mechanism, leading to weakening of the adherence of the neurosensory retina, which can cause the macular hole to progress to a retinal detachment $[3,6]$.

Macular holes associated with Best disease are rare. We found 9 reports in the literature, 3 of which were associated with retinal detachment [3, 7, 8]. Schachat et al. [7] reported performing vitrectomy and fluid-air exchange, with postoperative prone positioning and laser photocoagulation around the hole with reattachment of the retina and improvement in vision from hand movements to $1.00 \log$ MAR. Soliman [3] reported 3 cases, one of which had similar operation and visual outcome. Glacet-Bernard and Coscas [8] reported successful reattachment with pneumatic retinopexy and postoperative laser photocoagulation.

Our patient had an abnormally adherent gelatinous vitreous, which we think could be a feature of Best dystrophy. He showed good visual and anatomical outcome of his retinal detachment and macular hole after undergoing phacovitrectomy with silicone oil tamponade followed by silicone oil removal with ILM peel $>1$ year after the initial operation. The decision was made to use silicone oil as tamponade, instead of air or gas, especially as the macular hole was quite large, and there was difficulty removing the posterior hyaloid at the first operation.

After silicone oil insertion, the retina reattached and the macular hole underwent type 2 closure [9], i.e., the edges of the macular hole were re-apposed to the RPE but with a residual defect in the photoreceptor layer. This defect reduced in size with time under silicone oil (Fig. $2 \mathrm{~b}, \mathrm{c}$ ). The ILM was peeled at the time of silicone oil removal in order to achieve reduction in the photoreceptor layer defect or as close to type 1 closure as possible. Given that Best dystrophy results in loss of the central photoreceptors, we knew this patient would still have a residual defect. Despite this, the patient not only accomplished significant reduction in the foveal defect (from $1,102 \mu \mathrm{m}$ to $254 \mu \mathrm{m}$ ), which would still be a type 2 closure [9], but he had improvement in visual acuity from $1.00 \log$ MAR to $0.6 \log$ MAR.

\section{Conclusion}

Macular holes with Best disease are rare and are thought to be due to rupture of a cyst in the vitelliform stage or atrophy in later stages. It can be associated with a posterior pole detachment due to dysfunctional retinal pigment epithelium pump function. This case outlines that closure of the macular hole, flattening of the detachment, and improvement in visual acuity is possible with vitrectomy and ILM peeling. 


\section{Acknowledgement}

The authors would like to thank the staff at Worthing Hospital for the included images.

\section{Statement of Ethics}

This research was conducted ethically in accordance with the World Medical Association Declaration of Helsinki. The subject has provided written informed consent for the case to be published, including publication of images.

\section{Disclosure Statement}

The authors have no financial or proprietary interests in any of the products named. The authors have no financial or conflicting interests to disclose.

\section{Funding Sources}

No financial support or research funding was received for this study.

\section{Author Contributions}

Shamfa Peart: conception of work, data analysis and interpretation, drafting and critical revision, final approval. Amoy Ramsay: conception of work, data analysis and acquisition, drafting of work. Assad Khan: acquisition of data, revision. Tony Leong: conception of work, data acquisition. Patel Gordon-Bennett: conception of work, data acquisition, revision, final approval. The paper was presented as a poster at the British and Eire Association of Vitreoretinal Surgeons' Annual Meeting, Durham, United Kingdom, November 2-3, 2017.

\section{References}

1 Thu T, Chan WM, Dung D, Huan P, Lam DS. A large macular hole in a young patient with Best's disease. Clin Exp Ophthalmol. 2003 Dec;31(6):539-40.

2 Mandal S, Basu S. Giant macular hole with Best's disease in a young boy. Clin Exp Optom. 2012 Nov;95(6):643-5.

3 Soliman MM. Vitelliform macular dystrophy: a cause of macular holes with retinal detachments. Eye (Lond). 1994;8(Pt 4):484-7.

4 Goldberg N, Freund KB. Progression of an acquired vitelliform lesion to a full-thickness macular hole documented by eye-tracked spectral-domain optical coherence tomography. Arch Ophthalmol. 2012 Sep;130(9):1221-3.

5 Mehta M, Katsumi O, Tetsuka S, Hirose T, Tolentino FI. Best's macular dystrophy with a macular hole. Acta Ophthalmol (Copenh). 1991 Feb;69(1):131-4.

6 Liu J, Xuan Y, Zhang Y, Liu W, Xu G. Bilateral macular holes and a new onset vitelliform lesion in Best disease. Ophthalmic Genet. 2017 Jan-Feb;38(1):79-82.

7 Schachat AP, de la Cruz Z, Green WR, Patz A. Macular hole and retinal detachment in Best's disease. Retina. 1985;5(1):22-5.

8 Glacet-Bernard A, Coscas G. Full-thickness macular hole and retinal detachment complicating Best's disease. Eur J Ophthalmol. 1993 Jan-Mar;3(1):53-4.

9 Kang SW, Ahn K, Ham DI. Types of macular hole closure and their clinical implications. Br J Ophthalmol. 2003 Aug;87(8):1015-9. 


\section{Case Reports in Ophthalmology}

\begin{tabular}{l|l}
\hline Case Rep Ophthalmol 2019;10:221-226 \\
\hline DOI: 10.1159/000501845 & $\begin{array}{l}\text { ○ 2019 The Author(s). Published by S. Karger AG, Basel } \\
\text { www.karger.com/cop }\end{array}$ \\
\hline
\end{tabular}

Peart et al.: Large, Spontaneous Macular Hole with Posterior Pole Detachment in a Patient with Best Vitelliform Macular Dystrophy
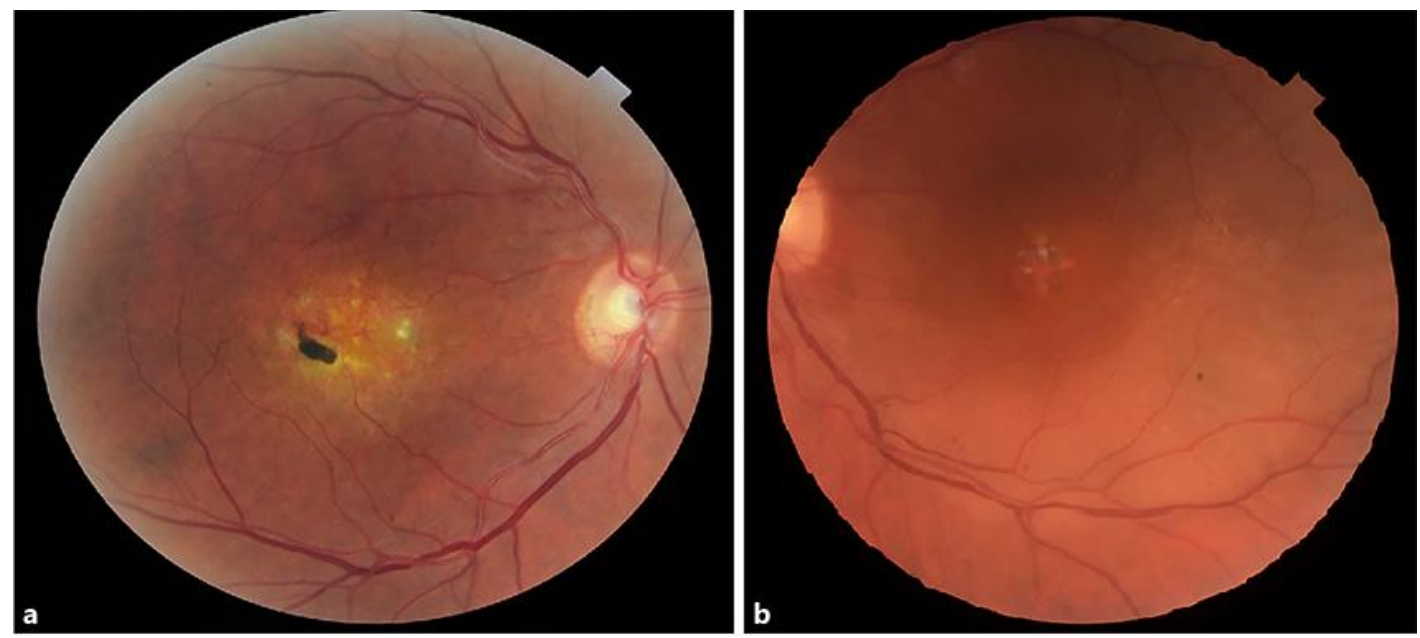

Fig. 1. Colour fundus photographs depicting pigmentary changes at the macula in the right eye (a) and pigmentary changes, macular hole, and a shallow posterior pole detachment in the left eye (b).

\begin{tabular}{|l|l|}
\hline \multicolumn{2}{|c|}{ Left Visual Acuity (Log MAR) } \\
Preoperative & 1.0 \\
Postop 3 months & 1.0 pinhole 0.8 \\
Postop 11 months & 1.0 pinhole 0.6 \\
\hline $\begin{array}{l}1 \text { month } \\
\text { Post ROSO, ILM peel } \\
\text { (done 12-9-17) }\end{array}$ \\
\begin{tabular}{l} 
(1) \\
\hline
\end{tabular} \\
\hline
\end{tabular}

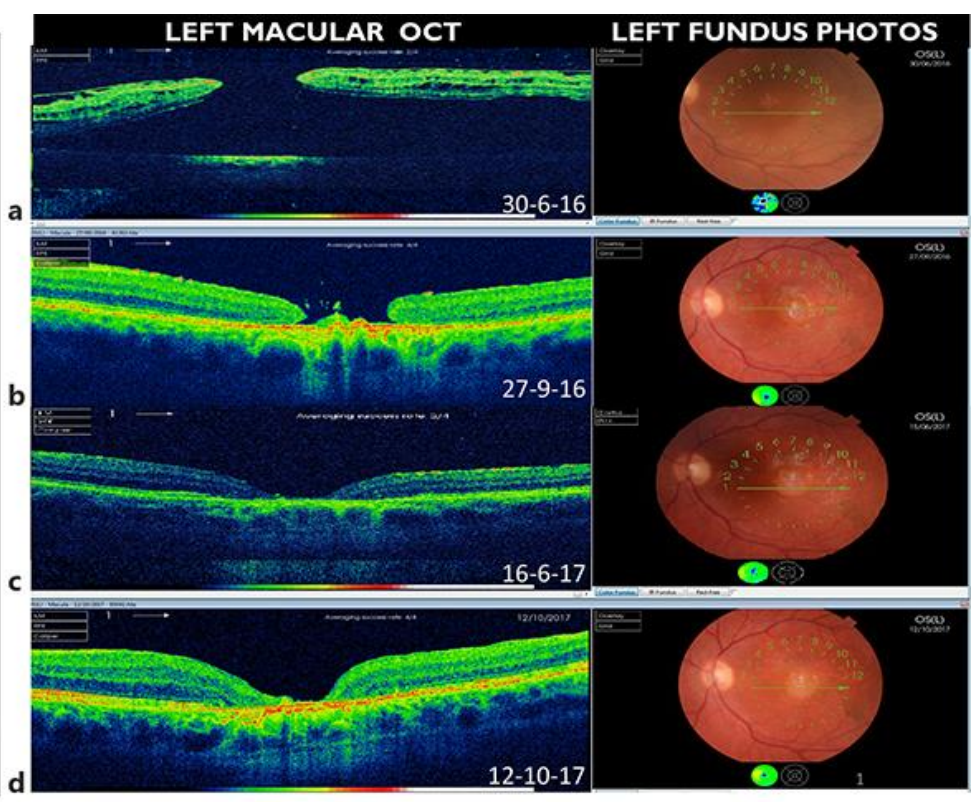

Fig. 2. Optical coherence tomography and fundus photographs depicting preoperative and postoperative status. a Preoperative. $\mathbf{b}$ Three months postoperative. c Eleven months postoperative. $\mathbf{d}$ One month postremoval of silicone oil (14.5 months post-initial operation). 


\section{Case Reports in Ophthalmology}

Peart et al: Large, Spontaneous Macular Hole with Posterior Pole Detachment in a Patient with Best Vitelliform Macular Dystrophy

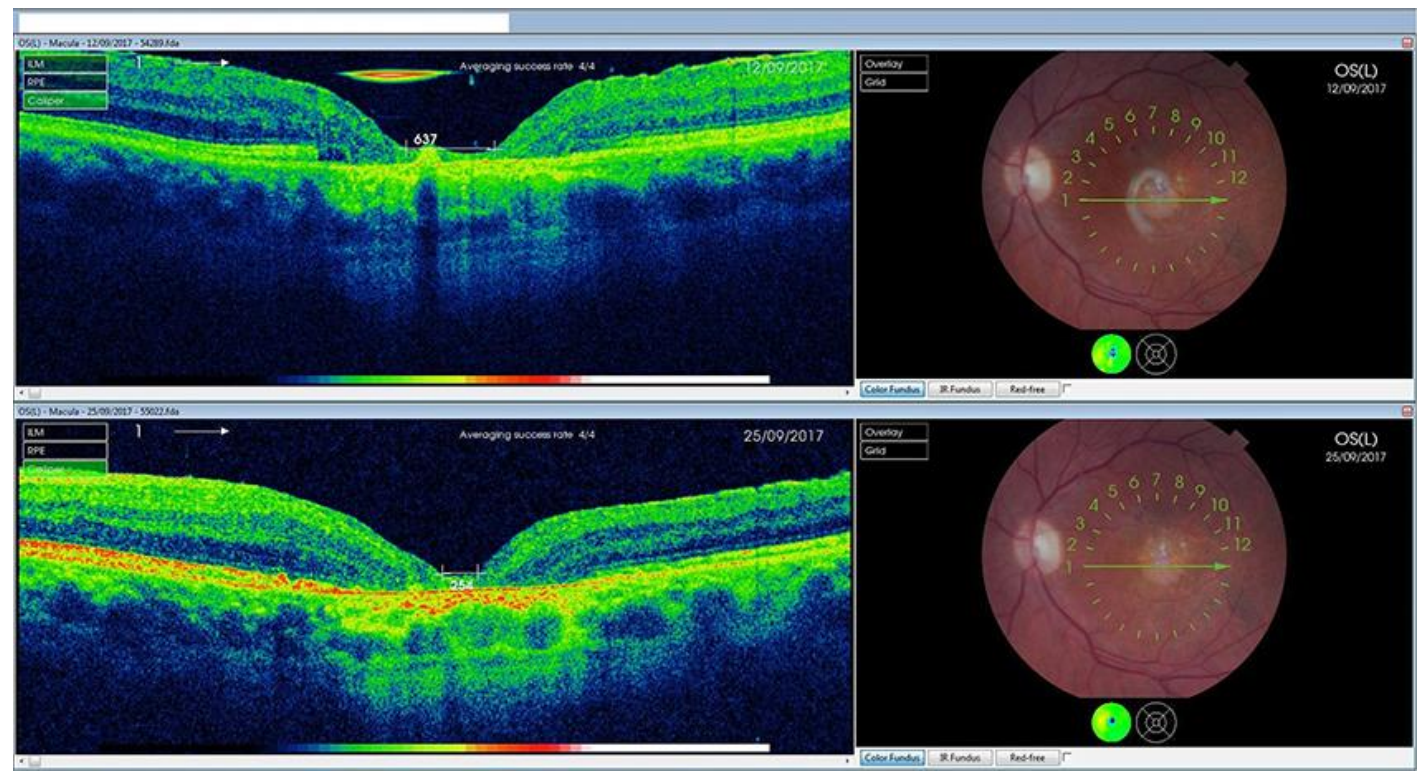

Fig. 3. Optical coherence tomography and fundus photographs showing reduction in foveal defect after oil removal and ILM peel. 